\title{
Anger is associated with subclinical atherosclerosis in low SES but not in higher SES men and women : the Cardiovascular Risk in Young Finns Study
}

\section{Merjonen, Päivi}

2008

Merjonen, P , Pulkki-Råback , L , Puttonen , S , Keskivaara , P , Juonala , M , Telama , R , Viikari , J , Raitakari , O T \& Keltikangas-Järvinen , L 2008 , ' Anger is associated with subclinical atherosclerosis in low SES but not in higher SES men and women : the Cardiovascular Risk in Young Finns Study ' , Journal of Behavioral Medicine , vol. 31 , no. 1 , pp. 35-44 . https://doi.org/10.1007/s10865-007-9131-6

http://hdl.handle.net/10138/27269

https://doi.org/10.1007/s10865-007-9131-6

publishedVersion

Downloaded from Helda, University of Helsinki institutional repository.

This is an electronic reprint of the original article.

This reprint may differ from the original in pagination and typographic detail.

Please cite the original version. 


\title{
Anger is associated with subclinical atherosclerosis in low SES but not in higher SES men and women. The Cardiovascular Risk in Young Finns Study
}

\author{
Päivi Merjonen · Laura Pulkki-Råback • \\ Sampsa Puttonen · Pertti Keskivaara - Markus Juonala • \\ Risto Telama · Jorma Viikari · Olli T. Raitakari · \\ Liisa Keltikangas-Järvinen
}

Accepted: August 30, 2007 / Published online: 17 October 2007

(C) Springer Science+Business Media, LLC 2007

\begin{abstract}
We investigated the associations of anger and cynicism with carotid artery intima-media thickness (IMT) and whether these associations were moderated by childhood or adulthood socioeconomic status (SES). The participants were 647 men and 893 women derived from the population-based Cardiovascular Risk in Young Finns Study. Childhood SES was measured in 1980 when the participants were aged 3-18. In 2001, adulthood SES, anger, cynicism, and IMT were measured. There were no associations between anger or cynicism and IMT in the entire population, but anger was associated with thicker IMT in participants who had experienced low SES in childhood. This association persisted after adjustment for a
\end{abstract}

P. Merjonen - L. Pulkki-Råback · S. Puttonen .

P. Keskivaara · L. Keltikangas-Järvinen $(\bowtie)$

Department of Psychology, University of Helsinki, P.O. Box 9, 00014 Helsinki, Finland

e-mail: liisa.keltikangas-jarvinen@helsinki.fi

S. Puttonen

Finnish Institute of Occupational Health, Topeliuksenkatu 41 a

A, 00250 Helsinki, Finland

M. Juonala

The Centre of Applied and Preventive Cardiovascular Medicine, University of Turku, Turku 20520, Finland

R. Telama

LIKES Research Center, Keltavuokko 4 C, 40520 Jyvaskyla, Finland

J. Viikari

Department of Medicine, University of Turku, 20520 Turku, Finland

O. T. Raitakari

Department of Clinical Physiology, University of Turku, P.O.

Box 52, 20521 Turku, Finland host of cardiovascular risk factors. It is concluded that the ill health-effects of psychological factors such as anger may be more pronounced in individuals who have been exposed to adverse socioeconomic circumstances early in life.

Keywords Atherosclerosis - Intima-media thickness (IMT) - Cynicism · Anger · Hostility · Socioeconomic status (SES)

Anger and hostility are considered to be independent risk factors for coronary heart disease (CHD), although there are mixed findings (Rozanski et al. 1999; Smith et al. 2004). Hostility is a multidimensional construct consisting of components that are cognitive (cynical and mistrustful attitudes toward others), affective (anger), and behavioral (anger expressed outwardly, suppressed, or repressed), although the term hostility refers primarily to cynical attitudes (Greenglass and Julkunen 1989). Some components of hostility might be more harmful than others to the risk of atherosclerosis. Recently studies have shown an association between incident CHD events and the experience and expression of anger (Chang et al. 2002; Kawachi et al. 1996; Williams et al. 2000), which can be considered as representing the affective and behavioral components of hostility.

Carotid intima-media thickness (IMT) measured by ultrasound is a sensitive marker of subclinical atherosclerosis which is a preclinical state of CHD. IMT is related to vascular risk factors, the extent of coronary atherosclerosis, and the occurrence of coronary events (Bots et al. 1997; Burke et al. 1995; Heiss et al. 1991). The advantage of the ultrasound measurement of IMT is that it can be safely applied to asymptomatic people, enabling studies of 
atherosclerosis among healthy young people, who do not yet have clinically manifest disease. Exposure to risk factors in youth is associated with increased IMT in adulthood (Raitakari et al. 2003).

Anger and hostile attitudes have been shown to be associated with IMT cross-sectionally (Bleil et al. 2004; Everson-Rose et al. 2006; Knox et al. 2000; Matsumoto et al. 1993). In prospective studies both anger (Matthews et al. 1998; Räikkönen et al. 2004) and cynical hostility (Julkunen et al. 1994; Pollitt et al. 2005) have been shown to predict higher IMT or progression of IMT. However, in one study hostile attitudes and suppression of anger did not predict progression of IMT (Räikkönen et al. 2004). In summary, the research on the relationship between anger or cynical hostility and IMT has produced mixed findings and has mainly concentrated on either clinical samples or middle-aged populations.

In addition to anger and cynicism, low socioeconomic status (SES), both in childhood and in adulthood, might be considered a risk factor for atherosclerosis (Ranjit et al. 2006; Rosvall et al. 2002). Low SES has also been shown to be associated with high hostility (Gallo and Matthews 2003). It has been suggested that SES might moderate the effects of psychosocial factors as predictors of atherosclerosis. For instance, a study by Lynch et al. (1998) showed that the effect of cardiovascular reactivity on atherosclerotic progression depended on SES, i.e. a stronger association was observed in individuals with low SES. Although SES might moderate the effect of anger and cynicism on atherosclerosis, only a few studies have explicitly examined the effect of participants' SES on that relationship. According to Mendes de Leon (1992) impatience/irritability and anger-out are associated with CHD in middle-aged low SES male patients. On the other hand, Eng et al. (2003) found that anger-out may be a protective factor for stroke and nonfatal myocardial infarction (MI) among male health professionals and they concluded that anger expression may have differential effects depending on the SES of study populations. In line with these studies, Mittleman et al. (1997) found that the risk of episodes of anger triggering the onset of nonfatal MI declined as the SES of patients increased, meaning that SES moderated the role of anger as a trigger of $\mathrm{MI}$ and that anger produced the greatest risk for low-SES individuals. Therefore, it is possible that anger and cynicism may produce differential atherosclerosis risk within subgroups of SES, although EversonRose et al. (2006) did not observe an interaction between cynical hostility and education level in predicting IMT among middle-aged women. According to our knowledge, there is no previous study on the interaction between anger or cynicism and SES on IMT in a young, population-based sample where SES is measured at different developmental stages. SES over the life-course may have important effects and it is not known whether SES at different developmental transitions affects the relationship between cynicism or anger and subclinical atherosclerosis.

The aim of the current study was to examine the relationships of cynicism and anger with carotid IMT and to determine whether these relationships were moderated by childhood and adulthood socioeconomic backgrounds. The sample used was nationally representative of Finnish young adults. We also tested whether these associations were independent of physiological risk factors, health-related behaviors, and lack of social support, all of which have been hypothesized to mediate the association between hostility and atherosclerosis (Smith et al. 2004).

\section{Methods}

\section{Participants}

Participants were 1,540 healthy men and women aged 24 39 in 2001 participating in the ongoing population-based research project called Cardiovascular Risk in Young Finns. In 1980, which was the baseline of the study, 4,320 participants in age cohorts of $3,6,9,12,15$, and 18 were invited to take part and 3,596 participated. To ensure a nationally representative sample, Finland was divided into five areas according to the location of the university cities with a medical school. In each area, urban and rural boys and girls were randomly selected from the Social Insurance Institution's population register, which covers the whole population of Finland (Åkerblom et al. 1991). The study was approved by local ethics committees and all participants gave written informed consent.

Two thousand two hundred and sixty four participants had valid IMT measures at the 21-year follow-up in 2001, of which 1,729 had valid psychological variables, 2,216 had SES data, 2,047 had data on health-related behaviors, 2,208 had complete data on physiological risk factors, and 1,732 had data on perceived social support. When these data were combined, the final sample consisted of those for which there was complete information on all study variables, a total of 1,540 participants (647 men and 893 women), which constitutes $68 \%$ of the total participants in 2001. It has been previously shown that those who dropped out during the 21-year follow-up period were slightly biased towards male gender, lower socio-economic status, and a more sedentary lifestyle than those who remained in the study, but no differences in physiological risk factor levels were found (Pulkki-Råback et al. 2005; Raitakari et al. 2003). 
Measures

Anger and cynicism

Anger (the suggested affective component of hostility) and cynicism (the suggested cognitive component of hostility) were measured in 2001 and were self-reported by the participants.

Anger was measured with a 7-item Irritability Scale of the Buss-Durkee Hostility Inventory (Buss and Durkee 1957). The Irritability Scale has been shown to correlate with the Spielberger State-Trait Anger Scale (Moreno et al. 1993). In addition, this scale has been suggested to be an adequate measure of anger by temperament research (Buss 1991). The items of the anger scale are "I lose my temper easily but get over it quickly", "I am irritated a great deal more than people are aware of", "It makes my blood boil to have somebody make fun of me", "Sometimes people bother me just by being around", "I often feel like a powder keg ready to explode", "I sometimes carry a chip on my shoulder", and "Lately, I have been kind of grouchy".

Cynicism was measured with a 7-item Cynicism scale derived from the Minnesota Multiphasic Personality Inventory (items 89, 93, 117, 124, 265, 316, and 319) (Comrey 1957, 1958). This scale shares five items with the 9-item cynical distrust factor derived by Greenglass and Julkunen (1989) and six items with the 13-item cynicism scale derived by Barefoot et al. (1989) from the CookMedley Hostility scale. The items of the cynicism scale are "It takes a lot of argument to convince most people of the truth", "I think most people would lie to get ahead", "Most people are honest chiefly through fear of being caught", "It is safer to trust nobody", "Most people will use somewhat unfair means to gain profit or an advantage rather than to lose it", "I think nearly anyone would tell a lie to keep out of trouble", and "Most people inwardly dislike putting themselves out to help other people". The cynicism scale has previously been associated with low social support (Keltikangas-Järvinen and Ravaja 2002) and cardiovascular risk factors, such as smoking, alcohol use and triglycerides (Keltikangas-Järvinen and Ravaja 2002; Pulkki et al. 2003). Each item was rated on a five-point scale, ranging from 1 (totally disagree) to 5 (totally agree), and the mean of each scale was calculated for only those who had responded to at least $50 \%$ of the items on the scale. Cronbach's $\alpha$ s for anger and cynicism scales were .76 and .79 , respectively.

These scales, even though not standardized measures of hostility, approximate the affective and cognitive components of hostility. In addition, these very scales (Keltikangas-Järvinen and Ravaja 2002; Pulkki et al. 2003) as well as similar constructs (Koskenvuo et al. 1988) have been used previously in a Finnish population-based studies of cardiovascular risk.

\section{Socioeconomic status}

Participant's childhood SES was measured by parents' years of education in 1980, using data of the more educated parent. Childhood SES was classified as low-SES $(<9$ years of education) and medium/high SES ( $\geq 9$ years of education). Likewise, adulthood SES was measured in 2001 as a dichotomous variable. However, because the general educational level has risen during the past two decades in Finland (Statistics Finland 2005), the cut-off point for lowSES was less than 13 years of education. In Finland, 9 years of education corresponds to having completed comprehensive school, and 13 years to having completed secondary education. Education was used as the marker of SES because the participants were fairly young at the measurement of their own adulthood SES (24-39 years), and therefore the youngest participants had not yet established their own occupational position in the labor market. In Finland, educational level correlates strongly with occupational position, and in a sub-analysis of the oldest participants of the present study $(n=1173$, mean age $=$ $35.8)$ education had a correlation of $.61(P<.001)$ with occupational status.

\section{Carotid intima-media thickness}

To assess carotid IMT, ultrasound studies were performed using Sequoia 512 ultrasound mainframes (Acuson, CA) with 13.0-MHz linear array transducers. The studies were performed between September 2001 and January 2002. The left common carotid artery was scanned by ultrasound technicians following a standardized protocol (Raitakari et al. 2003). A magnified image was recorded from an angle showing the greatest distance between lumen-intima interface and the media-adventitia interface. A moving scan with a duration of $5 \mathrm{~s}$, which included the beginning of the carotid bifurcation and the common carotid artery, was recorded and stored in digital format on optical discs for subsequent off-line analysis. The digitally stored scans were manually analyzed by a single reader blinded to participants' details. The analyses were performed using ultrasonic calipers. From the 5-s clip image, the best quality end-diastolic frame was selected (incident with the R-wave on a continuously recorded electrocardiogram). From this image, at least four measurements of common carotid far wall were taken approximately $10 \mathrm{~mm}$ proximal to the bifurcation to derive a mean carotid IMT. We have reported a $6.4 \%$ between-visit coefficient and a 5.2\% between-observer coefficient of variation in the IMT measurements (Raitakari et al. 2003). Common carotid 
artery and carotid bulb areas were scanned for the presence of plaques. Plaques were defined as a distinct area of the vessel wall protruding into the lumen $>50 \%$ of the adjacent intima-media layer. Wall regularity or echogenicity were not measured. None of subjects had plaques in the common carotid.

\section{Atherosclerosis risk factors}

The atherosclerosis risk factors below were measured in 2001. Health-related behaviors included smoking status (daily smoker versus non-smoker), heavy alcohol consumption, and physical activity. Heavy alcohol consumption was measured as how often alcohol was used with at least six portions at a time (one portion equals to $12 \mathrm{~g}$ ) ranging from 1 (once a year or never) to 6 (at least twice a week). Physical activity was measured with an index of intensity, frequency, and duration of physical activity (Telama et al. 2005). High scores on the Physical Activity Index (Cronbach's $\alpha=.76$ ) indicate high physical activity.

Physiological risk factors included body-mass index (BMI), cholesterol levels, and blood pressure. BMI was calculated as weight $(\mathrm{kg}) /$ height $^{2}\left(\mathrm{~m}^{2}\right)$. Measurements of lipid levels were taken in duplicate in the same laboratory. Standardized enzymatic methods were used for measuring the level of serum high-density lipoprotein (HDL) cholesterol, and low-density lipoprotein (LDL) cholesterol concentration was calculated according to the Friedewald formula (Friedewald et al. 1972). Blood pressure was measured with random zero sphygmomanometer in sitting position after $5 \mathrm{~min}$ rest. Readings to the nearest even number of millimeters of mercury were performed at least three times on each subject, and the average of these measurements was used in statistical analysis (Juonala et al. 2004).

Social support was assessed with the Perceived Social Support Scale-Revised consisting of 12 items (e.g., "My family always supports me when I need help") (Blumenthal et al. 1987). The participants rated the items on a 5-point scale ranging from totally disagree (1) to totally agree (5). The items were inversely coded, and the mean of 12 items was calculated to present social support received from family and friends, with high scores indicating low support. The Cronbach's $\alpha$ for the social support scale was .94 .

\section{Statistical analyses}

First we tested whether genders should be analyzed together or separately. This was done with triplicate interactions between cynicism or anger, gender and SES predicting IMT. The bivariate associations between all the study variables were examined with partial correlation analysis. The interaction effects between SES and cynicism or anger on IMT were tested with linear regression analysis with centered variables. When a significant interaction effect was found the population was split in two according to the moderator variable (SES) as recommended by Kraemer et al. (2001). Thus, subsequent analyses of the associations between cynicism or anger and IMT were performed separately within low and medium/high SES groups with multiple linear regression analysis adjusted for different blocks of atherosclerosis risk factors as follows: Model 1 included age and gender, Model 2 included age, gender, and the other SES variable as a covariate, Model 3 included age, gender, and health-related behaviors, Model 4 included age, gender, and physiological risk factors, Model 5 included age, gender, and social support, and Model 6 included all the covariates. Age and gender were used as covariates in all the analyses. Because the distribution was positively skewed, social support was log transformed before analyses. Statistical analyses were performed using SPSS 15.0.

\section{Results}

Gender differences and descriptives of the sample

The triplicate interactions concerning gender differences were found to be non-significant (all $P$ 's $>.10$ ). Thus, genders were analyzed together in subsequent analyses. Table 1 shows the descriptives of the sample.

Bivariate associations between anger, cynicism, risk factors, and IMT

Age- and gender-adjusted correlation coefficients are shown in Table 2. High cynicism and anger were associated with low adulthood SES, adverse health-related behaviors, high BMI and low social support ( $r$ s ranged between I.07। and I.27l, $P \mathrm{~s}<.05)$. In addition high cynicism correlated with low childhood SES $(r=1.061, P<.05)$. Low childhood SES was associated with smoking, low physical activity and low social support ( $r$ s ranged between I.05I and I.06l, $P \mathrm{~s}<$ $.05)$ and low adulthood SES with adverse health-related behaviors, higher BMI and less social support ( $r$ s ranged between I.06l and I.21I, $P$ s < .05). High IMT correlated with high LDL-cholesterol, BMI, systolic and diastolic blood pressure ( $r$ s ranged between .05 and $.17, P \mathrm{~s}<.05$ ). Cynicism, anger, and SES were not associated with IMT. The mediating role of anger in the association between childhood SES and IMT was also tested. The childhood SESIMT association without the mediator was $\beta=0.03$, 
Table 1 Characteristics of the study group $N=1,540$

\begin{tabular}{|c|c|c|c|}
\hline \multirow[b]{2}{*}{ Variable } & \multicolumn{3}{|c|}{$N=1,540$} \\
\hline & $n$ & $\%$ & Mean $(S D)$ \\
\hline Age in 2001 & & & $31.56(5.05)$ \\
\hline \multicolumn{4}{|l|}{ Gender } \\
\hline Men & 647 & 42.0 & \\
\hline Women & 893 & 58.0 & \\
\hline Cynicism $^{\mathrm{a}}$ & & & $2.70(0.69)$ \\
\hline Anger $^{\mathrm{a}}$ & & & $2.51(0.71)$ \\
\hline \multicolumn{4}{|l|}{ Childhood SES in $1980^{\mathrm{b}}$} \\
\hline Low & 507 & 32.9 & \\
\hline Medium and high & 1033 & 67.1 & \\
\hline \multicolumn{4}{|l|}{ Adulthood SES in $2001^{\mathrm{c}}$} \\
\hline Low & 412 & 26.8 & \\
\hline Medium and high & 1128 & 73.2 & \\
\hline \multicolumn{4}{|l|}{ Smoking } \\
\hline Non-smoker & 1225 & 79.5 & \\
\hline Smoker & 315 & 20.5 & \\
\hline Heavy alcohol consumption ${ }^{\mathrm{d}}$ & & & $2.53(1.37)$ \\
\hline Physical activity index ${ }^{\mathrm{e}}$ & & & $9.66(2.29)$ \\
\hline HDL-cholesterol (mmol/l) & & & $1.31(0.32)$ \\
\hline LDL-cholesterol (mmol/l) & & & $3.27(0.85)$ \\
\hline Body mass index $\left(\mathrm{kg} / \mathrm{m}^{2}\right)$ & & & $24.79(4.29)$ \\
\hline Systolic blood pressure (mmHg) & & & $116.33(13.00)$ \\
\hline Diastolic blood pressure (mmHg) & & & $70.50(10.59)$ \\
\hline Social support ${ }^{\mathrm{a}}$ & & & $1.79(0.78)$ \\
\hline IMT (mm) & & & $0.58(0.09)$ \\
\hline
\end{tabular}

${ }^{a}$ Cynicism, anger, and social support have possible values ranging from 1 to 5

${ }^{\mathrm{b}}$ Low versus medium and high childhood SES = under 9 years versus 9 years or more of education

${ }^{c}$ Low versus medium and high adulthood SES = under 13 years versus 13 years or more of education

${ }^{\mathrm{d}}$ Heavy alcohol consumption has values ranging from 1 ( = once a year or never) to 6 (=at least twice a week)

e Physical activity index has values ranging from 5 to 16

$P=.21$, and with anger as the mediator $\beta=0.03, P=.18$, thereby providing no evidence for mediation.

Interactions between cynicism or anger and SES in predicting IMT

Table 3 shows cynicism $\times$ SES and anger $\times$ SES interactions in predicting IMT. Adulthood SES did not have any interactive effects with hostility in predicting IMT. The interactions were additionally re-conducted using a threegrade adulthood SES measure ( $1=$ comprehensive school, 2 = secondary education, 3 = academic education), which did not show any interactive effects in predicting IMT (data not shown). Also, childhood SES did not moderate the relationship between cynicism and IMT. However, the childhood SES $\times$ anger interaction was significant $(t=$ $-3.29, P=.001)$.

As shown in Fig. 1 and Table 4, anger was positively associated with IMT in those with low childhood SES, whereas anger was not associated with IMT in those having medium/high childhood SES. Results of separate analyses for the low and medium/high childhood SES groups are shown in Table 4. Among participants with low childhood SES, higher anger was associated with higher IMT ( $B=0.02, t=3.73, P<.001)$. This means that a one-point increase in anger corresponds to a $0.02 \mathrm{~mm}$ increase in IMT among low-childhood-SES individuals. This association remained significant after separate adjustment for the participants' adulthood SES, health-related behaviors, physiological risk factors, and social support. Anger also remained significantly associated with IMT after simultaneous adjustment for these atherosclerosis risk factors among low-childhood-SES participants $(B=0.02$, $t=3.39, P=.001)$. If participants had higher childhood SES, anger was not associated with IMT $(B=-0.00 t=$ $-0.98, P=.33)$. In an additional analysis where only anger was used as an explanatory factor of IMT, anger explained $1.6 \%$ of the variance in IMT among low-childhood-SES participants.

\section{Discussion}

The present findings emphasize the modifying role of childhood SES in the association between anger and preclinical atherosclerosis. In the present sample of young men and women, anger, cynicism or SES had no independent associations with atherosclerosis in all participants, but within the subgroup of low-childhood-SES individuals, anger was associated with higher IMT. Among those who had grown up in a low-SES environment, carotid artery IMT was the highest in individuals who reported higher experience of anger. Experiences of anger were not related to carotid artery IMT in the higher-childhood-SES group. In low-SES participants, the anger-IMT association was independent of, or only marginally attenuated by, participant's adulthood SES, adverse health-related behaviors, physiological atherosclerosis risk factors, and lack of social support. The present study stresses that the behavioral risk factors for atherosclerosis might differ according to the participants' SES during developmental years in childhood.

The explanation for why anger, but not cynicism, was associated with atherosclerosis among low-childhood-SES individuals is unknown and requires further investigation. However, previous research provides more consistent 
Table 2 Age- and gender-adjusted partial correlations between the study variables

\begin{tabular}{|c|c|c|c|c|c|c|c|c|c|c|c|c|c|}
\hline & 1 & 2 & 3 & 4 & 5 & 6 & 7 & 8 & 9 & 10 & 11 & 12 & 13 \\
\hline \multicolumn{14}{|l|}{ 1. Cynicism } \\
\hline 2. Anger & $.39 * * *$ & & & & & & & & & & & & \\
\hline 3. Childhood SES & $-.06^{*}$ & -.05 & & & & & & & & & & & \\
\hline 4. Adulthood SES & $-.15 * * *$ & $-.07 *$ & $.20 * * *$ & & & & & & & & & & \\
\hline 5. Smoking & $.09 * * *$ & $.07 * *$ & $-.05 *$ & $-.21 * * *$ & & & & & & & & & \\
\hline 6. Alcohol consumption & $.12 * * *$ & $.08 * *$ & .04 & $-.08 * *$ & $.27 * * *$ & & & & & & & & \\
\hline 7. PAI & $-.07 * *$ & $-.11 * * *$ & $.05^{*}$ & $.12 * * *$ & $-.17 * * *$ & $.07 * *$ & & & & & & & \\
\hline 8. HDL-cholesterol & -.04 & -.01 & .00 & .04 & $-.08 * *$ & $.14 * * *$ & $.10^{* * *}$ & & & & & & \\
\hline 9. LDL-cholesterol & -.02 & .01 & -.02 & -.04 & .02 & -.03 & $-.08 * *$ & .00 & & & & & \\
\hline 10. BMI & $.11 * * *$ & $.11 * * *$ & -.02 & $-.06^{*}$ & -.01 & $.05 *$ & $-.05^{*}$ & $-.24 * * *$ & $.22 * * *$ & & & & \\
\hline 11. SBP & .03 & .01 & -.04 & .01 & $-.07 * *$ & .05 & $.05^{*}$ & -.01 & $.07 * *$ & $.32 * * *$ & & & \\
\hline 12. DBP & .05 & .01 & .02 & .01 & $-.09 * *$ & $.08 * *$ & .04 & $-.05^{*}$ & $.11 * * *$ & $.30 * * *$ & $.67 * * *$ & & \\
\hline 13. Social support & $.25^{* * *}$ & $.27 * * *$ & $-.06^{*}$ & $-.09 * * *$ & -.02 & -.01 & $-.11 * * *$ & -.03 & .01 & $.05^{*}$ & .03 & .05 & \\
\hline 14. IMT & -.01 & .04 & .03 & -.01 & .01 & .04 & .03 & -.02 & $.05^{*}$ & $.17 * * *$ & $.14 * * *$ & $.14 * * *$ & -.00 \\
\hline
\end{tabular}

PAI = Physical Activity Index, HDL = high-density lipoprotein, $\mathrm{LDL}=$ low-density lipoprotein, $\mathrm{BMI}=$ body mass index, $\mathrm{SBP}=$ systolic blood pressure, DBP = diastolic blood pressure, IMT = intima-media thickness

$* P<.05, * * P<.01, * * * P<.001$

evidence for the importance of anger in CHD than for hostility (Strike and Steptoe 2004). For example, anger may act as a trigger of MI (Mittleman et al. 1997). Anger might also be more strongly related to physiological reactivity than cynicism. In laboratory situations anger-provoking tasks are likely to elicit a positive association between hostility and cardiovascular reactivity (Suarez and Williams 1990), which is associated with IMT (Treiber et al. 2003). Thus, physiological reactivity may be a pathway whereby anger is associated with IMT.

Interestingly, childhood SES, but not adulthood SES, modified the association between anger and subclinical atherosclerosis. This was true, even though childhood and adulthood SES measures correlated with each other. This supports the idea that early life environment may have long-lasting effects, although these effects may be mediated by later life factors (Power et al. 1998). In the present sample, it has been found that a stressful home environment in childhood had more severe effects on cardiovascular risk factors (insulin resistance syndrome) in individuals coming from low-SES rather than high-SES families (Pulkki et al. 2003). The present findings thus extend our previous results and suggest that individuals with low childhood-SES may be more vulnerable to psychosocial stressors than their higher-SES counterparts. Low-SES individuals have less access to financial, human, and social capital, a fact which affects children's development and later health (Bradley and Corwyn 2002; Repetti et al. 2002). Gallo and Matthews (2003) have suggested in their reserve capacity model that low-SES individuals have fewer resources for managing stress and, as such, are more vulnerable to negative emotion and cognition. Thus, according to this model, anger has more detrimental effects on cardiovascular health in low-SES individuals because they are less equipped to deal with their negative emotionality.

It is also possible that childhood SES, instead of adult SES, was an important moderator in these relationships due to the young age of the sample and therefore relative recency of the childhood environment. For these young adults, their childhood SES probably accounts for most of their lifetime SES exposure. It is possible that the same pattern of findings would not be found in an older sample, in which adult SES factors may be more important determinants of health. The Cardiovascular Risk in Young Finns study will enable examination of this issue in future followup examinations, as participants get older.

The present results suggest that other atherosclerosis risk factors, such as health-related behaviors and lack of social support, contributed only minimally to the association between anger and IMT among low-childhood-SES individuals. Therefore, the mechanism linking anger to atherosclerosis remains unclear. Exaggerated cardiovascular and neuroendocrine reactivity and slower recovery have been suggested as pathways through which emotional factors may associate with cardiovascular disease (Björntorp 1991; McEwen 2000; Smith et al. 2004). These same physiological disturbances have been associated with low SES (Gump et al. 1999; McEwen 2000). Further studies should examine, in population-based samples, whether 
Table 3 Results of linear regression analyses regressing carotid IMT on anger or cynicism, SES, and their interaction effects $(N=1,540)$

$\beta=$ standardized regression coefficient

${ }^{\mathrm{b}} B=$ unstandardized regression coefficient

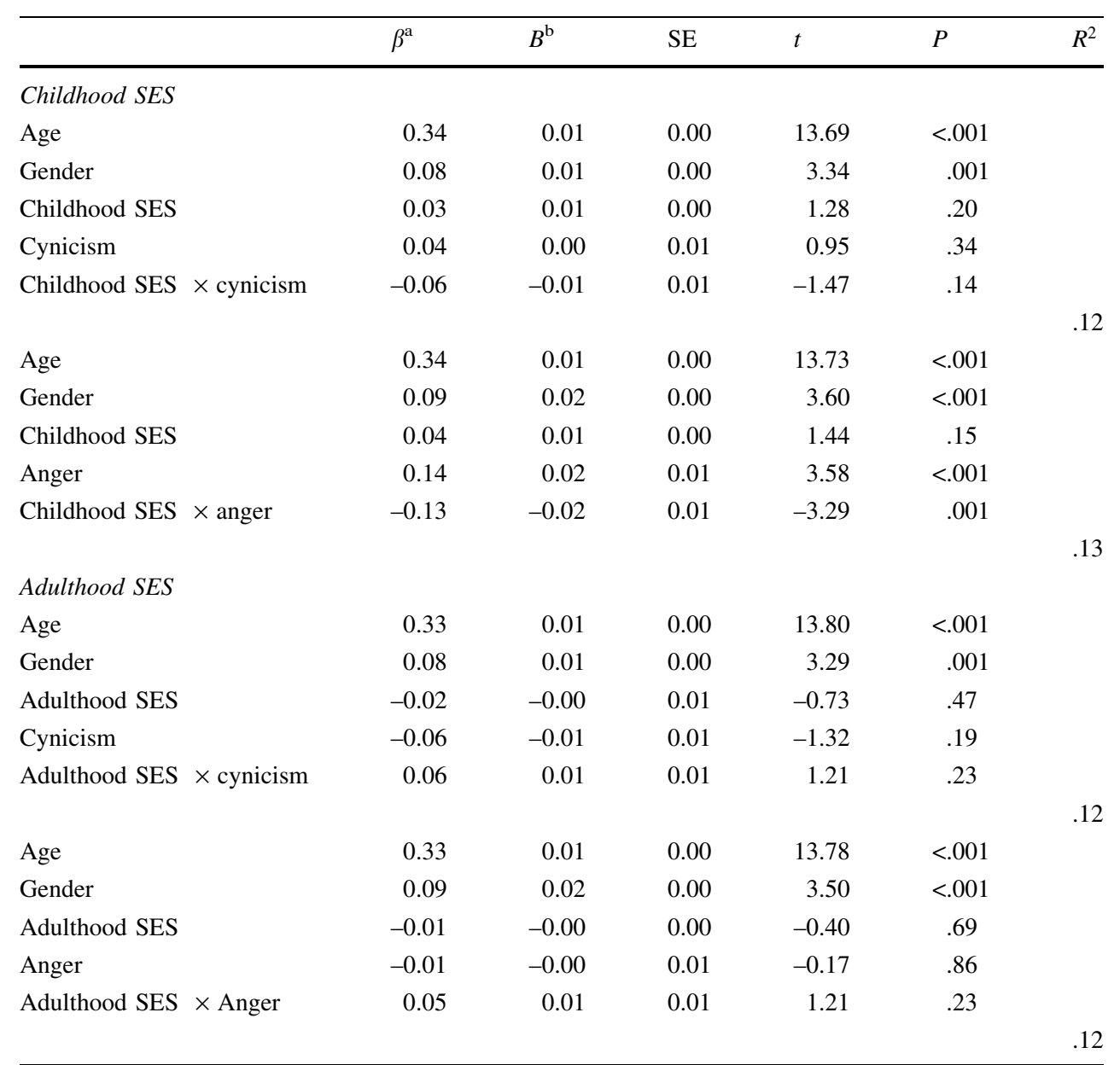

low-SES individuals with high levels of anger have different cardiovascular and neuroendocrine stress-responses compared to their higher-SES counterparts.

Among low-SES participants, anger explained only $1.6 \%$ of the variance in IMT. A one-point increase in mean anger, as measured on a five-point scale, corresponded to a $0.02 \mathrm{~mm}$ increase in IMT among low-childhood-SES individuals. This may seem rather small. However, a $0.16 \mathrm{~mm}$ difference in IMT has been shown to increase the risk of acute myocardial infarction by approximately $50 \%$ in subjects aged 55 years or more in the Rotterdam Study (Bots et al. 1997). Also, in the Atherosclerosis Risk in Communities Study, a $0.19 \mathrm{~mm}$ difference in mean IMT was shown to increase the risk of cardiovascular events by 69\% and 36\% among 45-64 years old women and men, respectively (Chambless et al. 1997). Individual differences in IMT are rather small among young and healthy people, and, therefore, in this healthy young sample even a small increase of IMT may be considered significant in terms of future risk.
Fig. 1 Age- and gender-adjusted regression lines of anger (range from $-2 S D$ to $+2 S D$ ) predicting carotid artery intima-media thickness (IMT) in participants with low and medium/high childhood socioeconomic status (SES) 
Table 4 Standardized $(\beta)$ and unstandardized $(B)$ regression coefficients of anger predicting IMT separately adjusted for different blocks of atherosclerosis risk factors for participants with low and medium/high childhood SES

\begin{tabular}{|c|c|c|c|c|c|c|c|c|c|c|c|c|}
\hline & \multicolumn{12}{|c|}{$N=1,540$} \\
\hline & \multicolumn{6}{|c|}{ Low childhood SES $N=507$} & \multicolumn{6}{|c|}{ Medium/high childhood SES $N=1,033$} \\
\hline & $R^{2 \dagger}$ & $\beta$ & $B$ & SE & $t$ & $P$-value & $R^{2 \dagger}$ & $\beta$ & $B$ & SE & $t$ & $P$-value \\
\hline Model $1^{\mathrm{a}}$ & .13 & 0.16 & 0.02 & 0.01 & 3.73 & $<.001$ & .13 & -0.03 & -0.00 & 0.00 & -0.98 & .33 \\
\hline Model $2^{\mathrm{b}}$ & .13 & 0.16 & 0.02 & 0.01 & 3.77 & $<.001$ & .13 & -0.03 & -0.00 & 0.00 & -1.08 & .28 \\
\hline Model $3^{\mathrm{c}}$ & .13 & 0.16 & 0.02 & 0.01 & 3.76 & $<.001$ & .13 & -0.03 & -0.00 & 0.00 & -0.98 & .33 \\
\hline Model $4^{\mathrm{d}}$ & .17 & 0.14 & 0.02 & 0.01 & 3.37 & .001 & .16 & -0.04 & -0.01 & 0.00 & -1.49 & .14 \\
\hline Model $5^{\mathrm{e}}$ & .13 & 0.16 & 0.02 & 0.01 & 3.68 & $<.001$ & .13 & -0.03 & -0.00 & 0.00 & -0.81 & .42 \\
\hline Model $6^{\mathrm{f}}$ & .18 & 0.15 & 0.02 & 0.01 & 3.39 & .001 & .16 & -0.04 & -0.01 & 0.00 & -1.34 & .18 \\
\hline \multicolumn{13}{|c|}{$R^{2}$ is for the whole model } \\
\hline \multicolumn{13}{|c|}{ a Model 1: Anger adjusted for age and gender } \\
\hline \multicolumn{13}{|c|}{ b Model 2: Anger adjusted for age, gender, and adulthood SES } \\
\hline \multicolumn{13}{|c|}{ c Model 3: Anger adjusted for age, gender, and health related behaviors (smoking, alcohol consumption, and physical activity index) } \\
\hline \multicolumn{13}{|c|}{$\begin{array}{l}\text { d Model 4: Anger adjusted for age, gender, and physiological risk factors (LDL-cholesterol, HDL-cholesterol, systolic blood pressure, diastolic } \\
\text { blood pressure, and BMI) }\end{array}$} \\
\hline \multicolumn{13}{|c|}{ e Model 5: Anger adjusted for age, gender, and social support } \\
\hline
\end{tabular}

The strengths of the study were that the participants were young and the sample was nationally representative of young Finnish adults and large enough to compare different gender and SES groups. We also were able to control for several factors that are plausible mechanisms relating anger to atherosclerosis. The current study demonstrated that in the presence of both low SES and high anger, effects on subclinical atherosclerosis become visible at a relatively young age, long before clinical manifestations of cardiovascular disease occur.

The cross-sectional design limits the conclusions that can be drawn regarding directionality. Specifically, although the participants in the present sample were apparently healthy, we cannot conclude whether it is anger that causes thickening of intima-media or vice versa. Another limitation was that only education was used as an indicator of SES. Education was used because it is usually fixed after young adulthood and is unlikely to be influenced by poor health later in life. Education also makes it possible to classify non-working people (e.g., home mothers) into socioeconomic groups.

\section{Conclusions}

The finding that it is childhood SES, but not adulthood SES, which modifies the association between anger and preclinical atherosclerosis, lends support to the idea that the health effects of anger are dependent on context and have their origins early in life. Our findings suggest that higher anger is related to higher IMT only among individuals with low-SES backgrounds, but the mechanisms underlying this association require further investigation.

Acknowledgments This study was supported by the Academy of Finland (Grant no. 111056, 209514, and 209518 (LK-J), and 77841 and 210283 (OTR)), The Finnish Foundation of Cardiovascular Research and The Yrjö Jahnsson Foundation.

\section{References}

Åkerblom, H. K., Uhari, M., Pesonen, E., Dahl, M., Kaprio, E. A., Nuutinen, E. M., Pietikäinen, M., Salo, M. K., Aromaa, A., Kannas, L., Keltikangas-Järvinen, L., Kuusela, V., Räsänen, L., Rönnemaa, T., Knip, M., Telama, R., Välimäki, I., Pyörälä, K., \& Viikari, J. (1991). Cardiovascular risk in young Finns. Annals of Medicine, 23, 35-39.

Barefoot, J. C., Dodge, K. A., Peterson, B. L., Dahlstrom, W. G., \& Williams, R. B., Jr. (1989). The Cook-Medley Hostility scale: Item content and ability to predict survival. Psychosomatic Medicine, 51, 46-57.

Björntorp, P. (1991). Visceral fat accumulation: The missing link between psychosocial factors and cardiovascular disease? Journal of Internal Medicine, 230, 195-201.

Bleil, M. E., McCaffery, J. M., Muldoon, M. F., Sutton-Tyrrell, K., \& Manuck, S. B. (2004). Anger-related personality traits and carotid artery atherosclerosis in untreated hypertensive men. Psychosomatic Medicine, 66, 633-639.

Blumenthal, J. A., Burg, M. M., Barefoot, J., Williams, R. B., Haney, T., \& Zimet, G. (1987). Social support, type A behavior, and coronary artery disease. Psychosomatic Medicine, 49, 331-340.

Bots, M. L., Hoes, A. W., Koudstaal, P. J., Hofman, A., \& Grobbee, D. E. (1997). Common carotid intima-media thickness and risk of stroke and myocardial infarction: The Rotterdam Study. Circulation, 96, 1432-1437. 
Bradley, R. H., \& Corwyn, R. F. (2002). Socioeconomic status and child development. Annual Review of Psychology, 53, 371-399.

Burke, G. L., Evans, G. W., Riley, W. A., Sharrett, A. R., Howard, G., Barnes, R. W., Rosamond, W., Crow, R. S., Rautaharju, P. M., \& Heiss, G. (1995). Arterial wall thickness is associated with prevalent cardiovascular disease in middle-aged adults: The Atherosclerosis Risk in Communities (ARIC) Study. Stroke, 26, 386-391.

Buss, A. H. (1991). The EAS theory of temperament. In Strelau, J. \& Angleitner, A. (Eds.), Explorations in temperament: International perspectives on theory and measurement (pp. 43-60). New York: Plenum Press.

Buss, A. H., \& Durkee, A. (1957). An inventory for assessing different kinds of hostility. Journal of Consulting Psychology, $21,343-349$.

Chambless, L. E., Heiss, G., Folsom, A. R., Rosamond, W., Szklo, M., Sharrett, A. R., \& Clegg, L. X. (1997). Association of coronary heart disease incidence with carotid arterial wall thickness and major risk factors: The Atherosclerosis Risk in Communities (ARIC) Study, 1987-1993. American Journal of Epidemiology, 146, 483-494.

Chang, P. P., Ford, D. E., Meoni, L. A., Wang, N. Y., \& Klag, M. J. (2002). Anger in young men and subsequent premature cardiovascular disease: The Precursors Study. Archives of Internal Medicine, 162, 901-906.

Comrey, A. L. (1957). A factor analysis of items on the MMPI hysteria scale. Educational and Psychological Measurement, 17, 586-592.

Comrey, A. L. (1958). A factor analysis of items on the MMPI paranoia scale. Educational and Psychological Measurement, 18, 99-107.

Eng, P. M., Fitzmaurice, G., Kubzansky, L. D., Rimm, E. B., \& Kawachi, I. (2003). Anger expression and risk of stroke and coronary heart disease among male health professionals. Psychosomatic Medicine, 65, 100-110.

Everson-Rose, S. A., Lewis, T. T., Karavolos, K., Matthews, K. A., Sutton-Tyrrell, K., \& Powell, L. H. (2006). Cynical hostility and carotid atherosclerosis in African American and white women: The Study of Women's Health Across the Nation (SWAN) Heart Study. American Heart Journal, 152, 982.e7-982.e13.

Friedewald, W. T., Levy, R. I., \& Fredrickson, D. S. (1972). Estimation of the concentration of low-density lipoprotein cholesterol in plasma, without use of the preparative ultracentrifuge. Clinical Chemistry, 18, 499-502.

Gallo, L. C., \& Matthews, K. A. (2003). Understanding the association between socioeconomic status and physical health: Do negative emotions play a role? Psychological Bulletin, 129, $10-51$.

Greenglass, E. R., \& Julkunen, J. (1989). Construct validity and sex differences in Cook-Medley Hostility. Personality and Individual Differences, 10, 209-218.

Gump, B. B., Matthews, K. A., \& Räikkönen, K. (1999). Modeling relationships among socioeconomic status, hostility, cardiovascular reactivity, and left ventricular mass in African American and white children. Health Psychology, 18, 140-150.

Heiss, G., Sharrett, A. R., Barnes, R., Chambless, L. E., Szklo, M., \& Alzola, C. (1991). Carotid atherosclerosis measured by B-mode ultrasound in populations: Associations with cardiovascular risk factors in the ARIC study. American Journal of Epidemiology, 134, 250-256.

Julkunen, J., Salonen, R., Kaplan, G. A., Chesney, M. A., \& Salonen, J. T. (1994). Hostility and the progression of carotid atherosclerosis. Psychosomatic Medicine, 56, 519-525.

Juonala, M., Viikari, J. S., Hutri-Kähönen, N., Pietikäinen, M., Jokinen, E., Taittonen, L., Marniemi, J., Rönnemaa, T., \& Raitakari, O. T. (2004). The 21-year follow-up of the Cardio- vascular Risk in Young Finns Study: Risk factor levels, secular trends and east-west difference. Journal of Internal Medicine, 255, 457-468.

Kawachi, I., Sparrow, D., Spiro, A. 3rd., Vokonas, P., \& Weiss, S. T. (1996). A prospective study of anger and coronary heart disease: The Normative Aging Study. Circulation, 94, 20902095.

Keltikangas-Järvinen, L., \& Ravaja, N. (2002). Relationships between hostility and physiological coronary heart disease risk factors in young adults: Moderating influence of perceived social support and sociability. Psychology \& Health, 17, 173-190.

Knox, S. S., Adelman, A., Ellison, R. C., Arnett, D. K., Siegmund, K., Weidner, G., \& Province, M. A. (2000). Hostility, social support, and carotid artery atherosclerosis in the National Heart, Lung, and Blood Institute Family Heart Study. American Journal of Cardiology, 86, 1086-1089.

Koskenvuo, M., Kaprio, J., Rose, R. J., Kesäniemi, A., Sarna, S., Heikkilä, K., \& Langinvainio, H. (1988). Hostility as a risk factor for mortality and ischemic heart disease in men. Psychosomatic Medicine, 50, 330-340.

Kraemer, H. C., Stice, E., Kazdin, A., Offord, D., \& Kupfer, D. (2001). How do risk factors work together? Mediators, moderators, and independent, overlapping, and proxy risk factors. American Journal of Psychiatry, 158, 848-856.

Lynch, J. W., Everson, S. A., Kaplan, G. A., Salonen, R., \& Salonen, J. T. (1998). Does low socioeconomic status potentiate the effects of heightened cardiovascular responses to stress on the progression of carotid atherosclerosis? American Journal of Public Health, 88, 389-394.

Matsumoto, Y., Uyama, O., Shimizu, S., Michishita, H., Mori, R., Owada, T., \& Sugita, M. (1993). Do anger and aggression affect carotid atherosclerosis? Stroke, 24, 983-986.

Matthews, K. A., Owens, J. F., Kuller, L. H., Sutton-Tyrrell, K., \& Jansen-McWilliams, L. (1998). Are hostility and anxiety associated with carotid atherosclerosis in healthy postmenopausal women? Psychosomatic Medicine, 60, 633-638.

McEwen, B. S. (2000). Allostasis and allostatic load: Implications for neuropsychopharmacology. Neuropsychopharmacology, 22, $108-124$.

Mendes de Leon, C. F. (1992). Anger and impatience/irritability in patients of low socioeconomic status with acute coronary heart disease. Journal of Behavioral Medicine, 15, 273-284.

Mittleman, M. A., Maclure, M., Nachnani, M., Sherwood, J. B., \& Muller, J. E. (1997). Educational attainment, anger, and the risk of triggering myocardial infarction onset. Archives of Internal Medicine, 157, 769-775.

Moreno, J. K., Fuhriman, A., \& Selby, M. J. (1993). Measurement of hostility, anger, and depression in depressed and nondepressed subjects. Journal of Personality Assessment, 61, 511-523.

Pollitt, R. A., Daniel, M., Kaufman, J. S., Lynch, J. W., Salonen, J. T., \& Kaplan, G. A. (2005). Mediation and modification of the association between hopelessness, hostility, and progression of carotid atherosclerosis. Journal of Behavioral Medicine, 28, 5364.

Power, C., Matthews, S., \& Manor, O. (1998). Inequalities in selfrated health: Explanations from different stages of life. Lancet, 351, 1009-1014.

Pulkki, L., Keltikangas-Järvinen, L., Ravaja, N., \& Viikari, J. (2003). Child-rearing attitudes and cardiovascular risk among children: Moderating influence of parental socioeconomic status. Preventive Medicine, 36, 55-63.

Pulkki, L., Kivimäki, M., Elovainio, M., Viikari, J., \& KeltikangasJärvinen, L. (2003). Contribution of socioeconomic status to the association between hostility and cardiovascular risk behaviors: A prospective cohort study. American Journal of Epidemiology, $158,736-742$. 
Pulkki-Råback, L., Elovainio, M., Kivimäki, M., Raitakari, O. T., \& Keltikangas-Järvinen, L. (2005). Temperament in childhood predicts body mass in adulthood: The Cardiovascular Risk in Young Finns Study. Health Psychology, 24, 307-315.

Räikkönen, K., Matthews, K. A., Sutton-Tyrrell, K., \& Kuller, L. H. (2004). Trait anger and the metabolic syndrome predict progression of carotid atherosclerosis in healthy middle-aged women. Psychosomatic Medicine, 66, 903-908.

Raitakari, O. T., Juonala, M., Kähönen, M., Taittonen, L., Laitinen, T., Mäki-Torkko, N., Järvisalo, M. J., Uhari, M., Jokinen, E., Rönnemaa, T., Åkerblom, H. K., \& Viikari, J. S. (2003). Cardiovascular risk factors in childhood and carotid artery intima-media thickness in adulthood: The Cardiovascular Risk in Young Finns Study. The Journal of American Medical Association, 290, 2277-2283.

Ranjit, N., Diez-Roux, A. V., Chambless, L., Jacobs, D. R., Jr., Nieto, F. J., \& Szklo, M. (2006). Socioeconomic differences in progression of carotid intima-media thickness in the Atherosclerosis Risk in Communities Study. Arteriosclerosis, Thrombosis, and Vascular Biology, 26, 411-416.

Repetti, R. L., Taylor, S. E., \& Seeman, T. E. (2002). Risky families: Family social environments and the mental and physical health of offspring. Psychological Bulletin, 128, 330-366.

Rosvall, M., Östergren, P. O., Hedblad, B., Isacsson, S. O., Janzon, L., \& Berglund, G. (2002). Life-course perspective on socioeconomic differences in carotid atherosclerosis. Arteriosclerosis, Thrombosis, and Vascular Biology, 22, 1704-1711.

Rozanski, A., Blumenthal, J. A., \& Kaplan, J. (1999). Impact of psychological factors on the pathogenesis of cardiovascular disease and implications for therapy. Circulation, 99, 21922217.

Smith, T. W., Glazer, K., Ruiz, J. M., \& Gallo, L. C. (2004). Hostility, anger, aggressiveness, and coronary heart disease: An interpersonal perspective on personality, emotion, and health. Journal of Personality, 72, 1217-1270.

Statistics Finland. (2005). Education statistics. Retrieved October 31, 2006, from http://www.stat.fi/tup/suoluk/suoluk_koulutus_en.html

Strike, P. C., \& Steptoe, A. (2004). Psychosocial factors in the development of coronary artery disease. Progress in Cardiovascular Diseases, 46, 337-347.

Suarez, E. C., \& Williams, R. B., Jr. (1990). The relationships between dimensions of hostility and cardiovascular reactivity as a function of task characteristics. Psychosomatic Medicine, 52, 558-570.

Telama, R., Yang, X., Viikari, J., Välimäki, I., Wanne, O., \& Raitakari, O. (2005). Physical activity from childhood to adulthood: A 21-year tracking study. American Journal of Preventive Medicine, 28, 267-273.

Treiber, F. A., Kamarck, T., Schneiderman, N., Sheffield, D., Kapuku, G., \& Taylor, T. (2003). Cardiovascular reactivity and development of preclinical and clinical disease states. Psychosomatic Medicine, 65, 46-62.

Williams, J. E., Paton, C. C., Siegler, I. C., Eigenbrodt, M. L., Nieto, F. J., \& Tyroler, H. A. (2000). Anger proneness predicts coronary heart disease risk: Prospective analysis from the Atherosclerosis Risk in Communities (ARIC) Study. Circulation, 101, 2034-2039. 